\title{
Frequency Interpolation of LOFAR Embedded Element Patterns Using Spherical Wave Expansion
}

\author{
M. J. Arts $\mathbb{D},,^{1,2}$ D. S. Prinsloo $\mathbb{D},,^{1,2}$ M. J. Bentum $\mathbb{D},{ }^{1,2}$ and A. B. Smolders $\mathbb{D}^{2}$ \\ ${ }^{1}$ ASTRON Netherlands Institute for Radio Astronomy, Dwingeloo, Netherlands \\ ${ }^{2}$ Eindhoven University of Technology, Eindhoven, Netherlands \\ Correspondence should be addressed to M. J. Arts; arts@astron.nl
}

Received 29 January 2021; Revised 19 April 2021; Accepted 3 June 2021; Published 16 June 2021

Academic Editor: Hervé Aubert

Copyright (c) 2021 M. J. Arts et al. This is an open access article distributed under the Creative Commons Attribution License, which permits unrestricted use, distribution, and reproduction in any medium, provided the original work is properly cited.

This paper describes the use of spherical wave expansion (SWE) to model the embedded element patterns of the LOFAR low-band array. The goal is to reduce the amount of data needed to store the embedded element patterns. The coefficients are calculated using the Moore-Penrose pseudoinverse. The Fast Fourier Transform (FFT) is used to interpolate the coefficients in the frequency domain. It turned out that the embedded element patterns can be described by only $41.8 \%$ of the data needed to describe them directly if sampled at the Nyquist rate. The presented results show that a frequency resolution of $1 \mathrm{MHz}$ is needed for proper interpolation of the spherical wave coefficients over the $80 \mathrm{MHz}$ operating frequency band of the LOFAR low-band array. It is also shown that the error due to interpolation using the FFT is less than the error due to linear interpolation or cubic spline interpolation.

\section{Introduction}

For the calibration of phased arrays, a priori knowledge of the embedded element patterns (EEPs) is needed. These patterns can be modeled by an analytic function and are often assumed to be the same for each antenna element. To improve the calibration, a full-wave electromagnetic simulation can be used to provide more accurate EEPs [1]. However, if the array is large, the amount of data also becomes large. A way to reduce the amount of data is the use of spherical wave expansion (SWE) to describe the EEPs.

The use of SWE related to radiation patterns is mainly seen in near-field to far-field transformations [2]. In [3], the use of SWE to describe the embedded element patterns of phased array antennas is described. One of the advantages of the use of SWE is that the correlation between two embedded element patterns can be computed efficiently [4].

In this paper, we will extend the use of spherical wave expansion with an interpolation technique in the frequency domain. This technique uses the Fast Fourier Transform (FFT) to interpolate the spherical wave coefficients in the frequency domain. These interpolated coefficients can be used to calculate the embedded element patterns at nonsimulated frequencies. The interpolation method can be used for phased arrays and Phased Array Feeds (PAFs). Application of PAF's can be found in radio astronomy [5-7] and future $5 \mathrm{G}$ communication systems [8]. The LOFAR radio telescope [9] will be used as a test case to verify our proposed concept.

The structure of this paper is as follows: Section 2 gives an overview of spherical wave expansion. Special attention is paid to the calculation of the coefficients which is done by using the Moore-Penrose pseudoinverse. The interpolation method is also discussed in this section. A description of the LOFAR radio telescope is given in Section 3. This section gives a general overview of the LOFAR system and a detailed description of the low-band antenna. The results are described in Section 4. Conclusions are given in Section 5 .

\section{Far-Field Spherical Wave Expansion}

2.1. Spherical Wave Functions. The electric field component of the far field of an antenna can be written as 


$$
\vec{E}\left(r, \widehat{u}_{r}\right)=\vec{e}\left(\widehat{u}_{r}\right) \frac{e^{-j \beta r}}{r},
$$

where $\beta$ is the free-space wave number, $r$ the distance between the antenna and the observation point, $\vec{e}\left(\widehat{u}_{r}\right)$ the normalized electric component of the far field, and $\widehat{u}_{\mathrm{r}}$ a unit vector pointing towards the observation point defined as

$$
\widehat{u}_{r}=\widehat{u}_{x} \sin \theta \cos \varphi+\widehat{u}_{y} \sin \theta \sin \varphi+\widehat{u}_{z} \cos \theta,
$$

where $\widehat{u}_{x}, \widehat{u}_{y}$, and $\widehat{u}_{z}$ are the unit vectors in the $x$-, $y$-, and $z$-direction, respectively. $\theta$ is the angle between $\widehat{u}_{r}$ and the $z$-axis measured from the $z$-axis and $\varphi$ is the angle between the $x$-axis and the projection of $\widehat{u}_{r}$ on the $x y$-plane measured from the $x$-axis (see Figure 1). The normalized electric far field can be expanded in terms of spherical wave functions:

$$
\vec{e}\left(\widehat{u}_{r}\right)=\sum_{n=1}^{\infty} \sum_{m=-n}^{n} \sum_{s=1}^{2} Q_{s m n} \vec{f}_{s m n}\left(\widehat{u}_{r}\right)
$$

where $\vec{f}_{s m n}\left(\widehat{u}_{r}\right)$ is the spherical function $(s, m, n)$ and $Q_{s m n}$ the corresponding coefficient. It is convenient to use a socalled "compressed index" $p$ defined as $[2, p .15]$

$$
p=2(n(n+1)+m-1)+s,
$$

to replace the indices $(s, m, n)$. The numbering scheme up to $n=2$ is shown in Table 1 .

Using the compressed index, equation (3) can be written as

$$
\vec{e}\left(\widehat{u}_{r}\right)=\sum_{p=1}^{\infty} Q_{p} \vec{f}_{p}\left(\widehat{u}_{r}\right)
$$
that

Each spherical wave function $\vec{f}_{p}\left(\widehat{u}_{r}\right)$ is normalized such

$$
\int_{\varphi=0}^{2 \pi} \int_{\theta=0}^{\pi}\left|\vec{f}_{p}\left(\widehat{u}_{r}(\theta, \varphi)\right)\right|^{2} \sin \theta \mathrm{d} \theta \mathrm{d} \varphi=Z_{F},
$$

where $Z_{F}$ is the wave impedance in free space $(\approx 377 \Omega)$. The spherical wave functions $\vec{f}_{p}\left(\widehat{u}_{r}(\theta, \varphi)\right)$ and $\vec{f}_{q}\left(\widehat{u}_{r}(\theta, \varphi)\right)$ are orthogonal with respect to each other:

$$
\int_{\varphi=0}^{2 \pi} \int_{\theta=0}^{\pi} \vec{f}_{p}\left(\widehat{u}_{r}(\theta, \varphi)\right) \cdot \vec{f}_{q}^{*}\left(\widehat{u}_{r}(\theta, \varphi)\right) \sin \theta \mathrm{d} \theta \mathrm{d} \varphi=0, \quad \text { for } p \neq q .
$$

Note that we used the notation $\widehat{u}_{r}(\theta, \varphi)$ to denote that $\widehat{u}_{r}$ depends on $\theta$ and $\varphi$ according to equation (2). The complex conjugate is denoted by $*$.

2.2. Calculation of the Coefficients $Q_{p}$. In practice, the summation over $n$ in (3) is limited to a finite number $N_{\max }$. By setting $n=m=N_{\max }$ and $s=2$ in (4), one can show that the maximum number of modes $N_{\text {modes }}$ should be at least

$$
\begin{aligned}
N_{\text {modes }} & =2\left(N_{\max }\left(N_{\max }+1\right)+N_{\max }-1\right)+2 \\
& =2 N_{\max }\left(N_{\max }+2\right),
\end{aligned}
$$

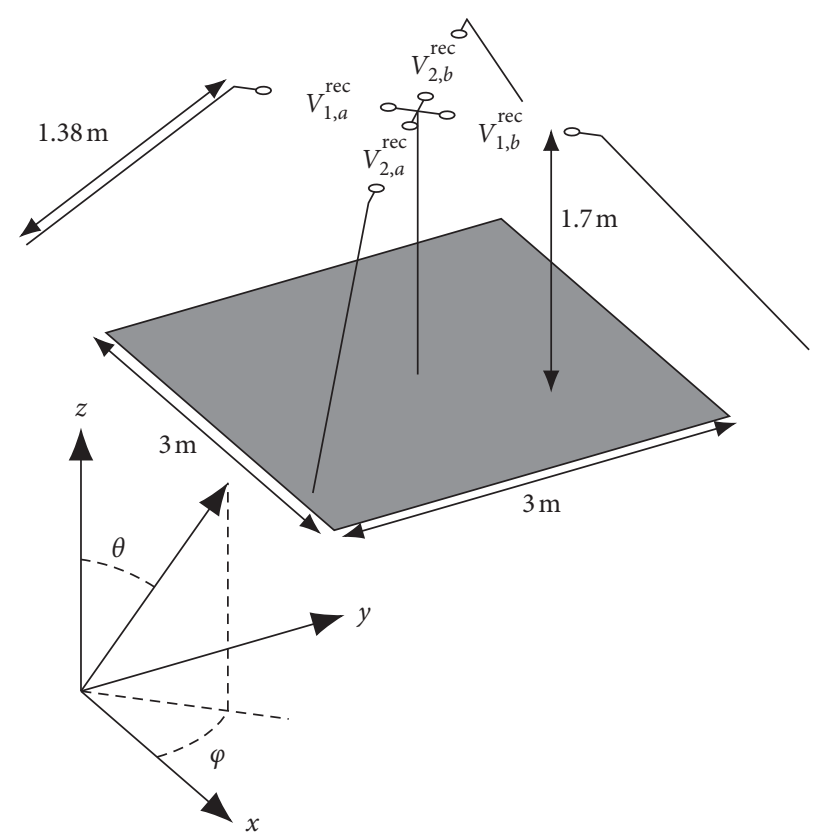

FIgURE 1: Simulation model of a low-band antenna.

TABLe 1: Numbering scheme of the compressed index $p$.

\begin{tabular}{ccccccccccccccccc}
\hline$p$ & 1 & 2 & 3 & 4 & 5 & 6 & 7 & 8 & 9 & 10 & 11 & 12 & 13 & 14 & 15 & 16 \\
\hline$s$ & 1 & 2 & 1 & 2 & 1 & 2 & 1 & 2 & 1 & 2 & 1 & 2 & 1 & 2 & 1 & 2 \\
$n$ & 1 & 1 & 1 & 1 & 1 & 1 & 2 & 2 & 2 & 2 & 2 & 2 & 2 & 2 & 2 & 2 \\
$m$ & -1 & -1 & 0 & 0 & 1 & 1 & -2 & -2 & -1 & -1 & 0 & 0 & 1 & 1 & 2 & 2 \\
\hline
\end{tabular}

$N_{\text {modes }}$ should be chosen such that the contribution of the higher-order modes $\left(>N_{\text {modes }}\right)$ can be neglected. As a rule of thumb, $N_{\max }$ will be chosen as [2, p. 17]

$$
N_{\max }=\beta r_{0}+10,
$$

which corresponds with

$$
N_{\text {modes }}=2\left(\beta r_{0}+10\right)\left(\beta r_{0}+12\right)=2 \beta^{2} r_{0}^{2}+44 \beta r_{0}+240 \text {, }
$$

where $r_{0}$ is the radius of the smallest sphere enclosing the antenna.

If the radiation pattern $\vec{e}\left(\widehat{u}_{r}\right)$ of an antenna is known in $N_{\text {dir }}$ directions and we want to describe it in $N_{\text {modes }}$ spherical modes, it can be written as a vector e with length $2 N_{\text {dir }}$ :

$$
\mathbf{e}=\left[\begin{array}{c}
e_{\theta}\left(\widehat{u}_{r, 1}\right) \\
\vdots \\
e_{\theta}\left(\widehat{u}_{r, N_{\mathrm{dir}}}\right) \\
e_{\varphi}\left(\widehat{u}_{r, 1}\right) \\
\vdots \\
e_{\varphi}\left(\widehat{u}_{r, N_{\mathrm{dir}}}\right)
\end{array}\right],
$$

where $e_{\theta}\left(\widehat{u}_{r, n}\right)$ and $e_{\varphi}\left(\widehat{u}_{r, n}\right)$ are, respectively, the $\theta$ - and $\varphi$-component of the normalized electric far field in the direction $\widehat{u}_{r, n}$ : 


$$
\vec{e}\left(\widehat{u}_{r, n}\right)=e_{\theta}\left(\widehat{u}_{r, n}\right) \widehat{u}_{\theta}+e_{\varphi}\left(\widehat{u}_{r, n}\right) \widehat{u}_{\varphi},
$$

where $\widehat{u}_{\theta}$ and $\widehat{u}_{\varphi}$ are the unit vector in $\theta$-and $\varphi$-direction, respectively. The far-field spherical vector functions for the first $N_{\text {modes }}$ modes specified in $N_{\text {dir }}$ directions can be written in a $2 N_{\text {dir }} \times N_{\text {modes }}$ matrix $\mathbf{F}$ :

$$
\mathbf{F}=\left[\begin{array}{ccc}
f_{\theta, 1}\left(\widehat{u}_{r, 1}\right) & \ldots & f_{\theta, N_{\text {modes }}}\left(\widehat{u}_{r, 1}\right) \\
\vdots & \ddots & \vdots \\
f_{\theta, 1}\left(\widehat{u}_{r, N_{\text {dir }}}\right) & \ldots & f_{\theta, N_{\text {modes }}}\left(\widehat{u}_{r, N_{\text {dir }}}\right) \\
f_{\varphi, 1}\left(\widehat{u}_{r, 1}\right) & \ldots & f_{\varphi, N_{\text {modes }}}\left(\widehat{u}_{r, 1}\right) \\
\vdots & \ddots & \vdots \\
f_{\varphi, 1}\left(\widehat{u}_{r, N_{\text {dir }}}\right) & \ldots & f_{\varphi, N_{\text {modes }}}\left(\widehat{u}_{r, N_{\text {dir }}}\right)
\end{array}\right] .
$$

The scalars $f_{\theta, p}\left(\widehat{u}_{r}\right)$ and $f_{\varphi, p}\left(\widehat{u}_{r}\right)$ are the $\stackrel{\theta}{\rightarrow}$ and $\varphi$-component of the spherical wave function $\vec{f}_{p}\left(\hat{u}_{r}\right)$, respectively:

$$
\vec{f}_{p}\left(\widehat{u}_{r}\right)=f_{\theta, p}\left(\widehat{u}_{r}\right) \widehat{u}_{\theta}+f_{\varphi, p}\left(\widehat{u}_{r}\right) \widehat{u}_{\varphi},
$$

where we used the compressed index $p$. The spherical mode coefficients $Q_{p}$ can be grouped in a vector $\mathbf{q}$ with length $N_{\text {modes }}$ :

$$
\mathbf{q}=\left[\begin{array}{c}
Q_{1} \\
\vdots \\
Q_{N_{\text {modes }}}
\end{array}\right] .
$$

Equation (5) can now be written as

$$
\mathbf{e}=\mathbf{F q} \text {. }
$$

The solution of equation (16) is given by [10]

$$
\mathbf{q}=\mathbf{F}^{\dagger} \mathbf{e}+\left(\mathbf{U}_{\mathbf{N}_{\text {modes }}}-\mathbf{F}^{\dagger} \mathbf{F}\right) \mathbf{w},
$$

where the operator ${ }^{\dagger}$ represents the Moore-Penrose pseudoinverse, $\mathbf{U}_{\text {Nmodes }}$ is an $N_{\text {modes }} \times N_{\text {modes }}$ identity matrix, and w is a column vector with length $N_{\text {modes }}$ in which elements can have arbitrary values. If $\mathbf{e}=\mathbf{F} \mathbf{F}^{\dagger} \mathbf{e}$, at least one solution exists. For the cases when no solutions exist, equation (17) gives the "closest" solution(s). Mathematically, these are the value or values of $\mathbf{q}$ for which the Euclidean norm $\|\mathbf{e}-\mathbf{F q}\|_{2}$ has its absolute minimum. If $\mathbf{F F}^{\dagger}=\mathbf{U}_{2 \mathbf{N} d i r}$ (with $\mathbf{U}_{2 \mathbf{N} d i r}$ a $2 N_{d i r} \times$ $2 N_{d i r}$ identity matrix), equation (16) has at least one solution for arbitrary e. This requirement is only fulfilled if the number of rows of $\mathbf{F}$ is equal to or less than its number of columns and $\mathbf{F}$ has a full (row) rank. In practical cases, the number of rows of $\mathbf{F}$ is larger than its number of columns, so equation (16) has no solution. In practice, the coefficients $Q_{p} \approx 0$ for $p>N_{\text {modes }}$, so the norm $\|\mathbf{e}-\mathbf{F q}\|_{2}$ will be very close to zero, and equation (17) can practically be considered as a solution of equation (16). If $\mathbf{F}^{\dagger} \mathbf{F}=\mathbf{U}_{\text {Nmodes }}$, there exists only one solution or value for which the norm $\|\mathbf{e}-\mathbf{F q}\|_{2}$ has its absolute minimum. This is only the case if the number of rows of $\mathbf{F}$ is equal to or larger than its number of columns and $\mathbf{F}$ has a full (column) rank. If $\mathbf{F}^{\dagger} \mathbf{F} \neq \mathbf{U}_{\text {Nmodes }}$, there exist infinite solutions or values for which the norm $\|\mathbf{e}-\mathbf{F q}\|_{2}$ has its absolute minimum. These solutions or values are parametric on the vector $\mathbf{w}$ in equation (17).
Figure 2 shows the rank of matrix $\mathbf{F}$ as a function of the number of columns (coefficients) for a different number of rows (angles).

The number of columns represents the number of spherical wave coefficients. The number of rows is directly related to the number of directions for which the far field is given. This relation is shown in Table 2. The number of rows is two times the product of the number of $\theta$ - and $\varphi$-angles for which the far field is given (note that the far field has a $\theta$ - and $\varphi$-component for each direction). The angles $\theta=0^{\circ}$ and $\theta=$ $180^{\circ}$ are not included because the spherical wave functions are not defined for these angles.

For an increasing number of columns (number of coefficients) for a given number of rows, the matrix $\mathbf{F}$ has full column rank until a certain number of columns. The highest number of columns where $\mathbf{F}$ has full column rank are denoted by $*$ in Figure 2. The column rank still increases if the number of columns is further increased. From a certain point where the number of columns is higher than the number of rows, $\mathbf{F}$ has a full row rank. These points are denoted by ${ }^{\circ}$ in Figure 2. For the cases when $\mathbf{F}$ has 5040 and 25776 rows, this number could not be determined because the size of $\mathbf{F}$ becomes too large.

Table 3 shows for which number of columns the matrix $\mathbf{F}$ has full row and column rank.

Table 3 shows, for example, that if matrix $\mathbf{F}$ has 288 rows ( $\theta$ - and $\varphi$-angles in steps of $\left.20^{\circ}\right)$ it has full column rank if the number of columns is less than or equal to 160 . This means that, for this case, equation (16) has one value for which the norm $\|\mathbf{e}-\mathbf{F q}\|_{2}$ has its absolute minimum if the number of coefficients $Q$ is chosen equal to or less than 160 . If the number of coefficients is 510 or more, there exists more than one vector $\mathbf{q}$ for which the norm has its absolute minimum. Note that there is no number of coefficients for which there exists one exact solution.

Figure 3 shows the condition number of the matrix $\mathbf{F}$ as a function of the number of columns for different numbers of rows. The point with the maximum number of columns where $\mathbf{F}$ has full column rank and the point with the minimum number of columns where $\mathbf{F}$ has full row rank are marked in the same way as in Figure 2.

One can see that the condition number remains below 10 until the point where $\mathbf{F}$ reaches full column rank. After this point, the condition number increases to values greater than $10^{15}$. A large condition number means that calculations involving $\mathbf{F}$ become numerically unstable; that is, a small variation of the far field e leads to a large variation of the computed coefficients $\mathbf{q}$. After $\mathbf{F}$ has reached full row rank, the condition number reduces.

The result of Figure 3 shows that, for solving equation (16) for a given number of directions where $\mathbf{e}$ is specified, the number of columns of $\mathbf{F}$ should be chosen such that the matrix has full column rank.

2.3. Interpolation. For frequency points in between the simulated frequencies, one has to interpolate the spherical wave coefficients and determine the embedded element patterns from these interpolated coefficients. 


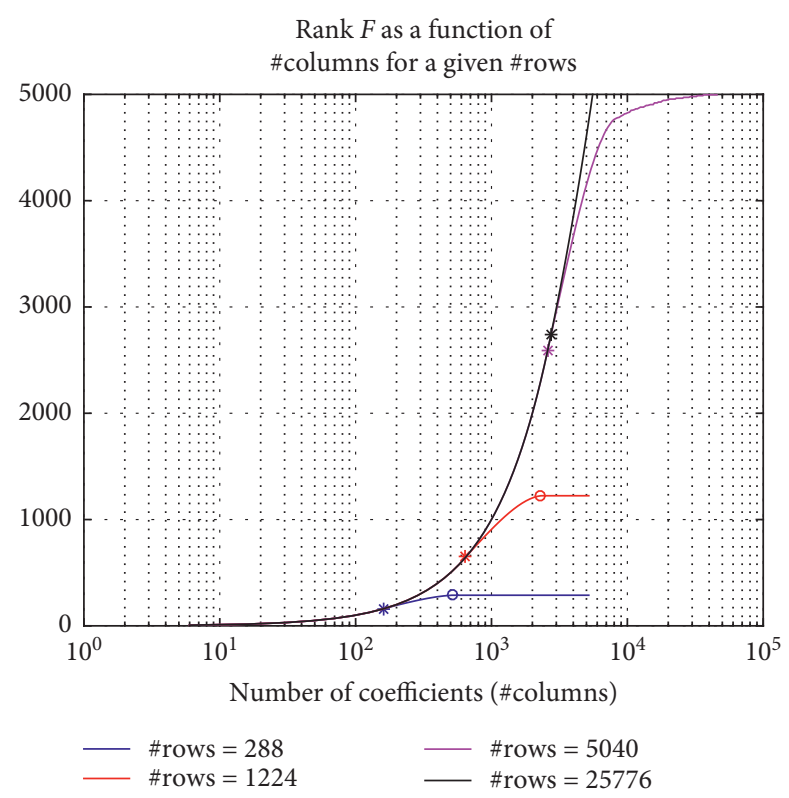

Figure 2: The rank of the matrix $\mathbf{F}$ as a function of the number of columns.

TABLE 2: Relation between the number of rows and directions for which the far field is given.

\begin{tabular}{lcccc}
\hline Number of rows & Number of $\theta$-angles & $\theta$-range & Number of $\varphi$-angles & $\varphi$-range \\
\hline 288 & 8 & $20^{\circ}$ to $160^{\circ}$ in steps of $20^{\circ}$ & 18 & $0^{\circ}$ to $340^{\circ}$ in steps of $20^{\circ}$ \\
1224 & 17 & $10^{\circ}$ to $170^{\circ}$ in steps of $10^{\circ}$ & 36 & $0^{\circ}$ to $350^{\circ}$ in steps of $10^{\circ}$ \\
5040 & 35 & $5^{\circ}$ to $175^{\circ}$ in steps of $5^{\circ}$ & 72 & $0^{\circ}$ to $355^{\circ}$ in steps of $5^{\circ}$ \\
25776 & 179 & $1^{\circ}$ to $179^{\circ}$ in steps of $1^{\circ}$ & 72 & $0^{\circ}$ to $355^{\circ}$ in steps of $5^{\circ}$ \\
\hline
\end{tabular}

TABle 3: Values for which the matrix $\mathbf{F}$ has full row and column rank.

\begin{tabular}{lcc}
\hline Number of rows & Full column rank up to & Full row rank from \\
\hline 288 & 160 columns & 510 columns \\
1224 & 646 columns & 2310 columns \\
5040 & 2590 columns & - \\
25776 & 2736 columns & - \\
\hline
\end{tabular}

Suppose that the spherical wave coefficients $Q_{n}(f)$ are calculated in steps $\Delta f$ between $f_{\text {min }}$ and $f_{\text {max }}\left(f_{\text {min }}\right.$ and $f_{\text {max }}$ included) which are both a multiple of the frequency step $\Delta f$, then $f_{\min }$ and $f_{\max }$ can be written as

$$
\begin{aligned}
& f_{\text {min }}=N_{1} \Delta f, \\
& f_{\text {max }}=N_{2} \Delta f .
\end{aligned}
$$

The number of frequencies is $N_{2}-N_{1}+1$. If the bins of the FFT are numbered from 0 to $M-1$, the values of $Q_{n}(f)$ are stored in the bins as shown in Table 4.

The bins $(M / 2)$ to $M-1$ represent the negative frequencies. In these bins, the complex conjugate of the coefficients (denoted by ${ }^{*}$ in Table 4 ) at the corresponding positive frequency is stored. The value of $M$ must be larger than $2 N_{2}+2$ and should be a power of 2 for an FFT. The spherical wave coefficients can be transformed to the time domain by using the inverse FFT:

$$
q_{n}(m \Delta t)=\frac{1}{M} \sum_{k=0}^{M-1} Q_{n}(k \Delta f) e^{j(2 \pi k m / M)} .
$$

Because $Q_{n}((M-k) \Delta f)=Q_{n}(-k \Delta f)=Q_{n}^{*}(k \Delta f)$, the time domain version of the spherical wave coefficient $q_{n}(m \Delta t)$ is a real function.

The length of the time domain window of the spherical wave coefficient is given by $T=(1 / \Delta f)$. The time step is given by $\Delta t=1 /(M \Delta f) \cdot q_{n}(m \Delta t)$ will decay to zero because the time domain response of an antenna element also decays to zero. If $T$ is large enough, the values of $q_{n}(m \Delta t)$ can be neglected for larger values of $m$. The value of $T$, for which this happens, determines the largest frequency step $\Delta f$ that can be used for interpolation; that is, the sampling in the frequency domain is too coarse and aliasing in the time domain will happen if $T$ is large enough. Due to the aliasing in the time domain, $q_{n}(m \Delta t)$ does not decay to zero for large values of $m$.

If the value of $T$ is large enough to neglect the effect of aliasing in the time domain, the spherical wave coefficients can be interpolated in the frequency domain by padding $q_{n}(m \Delta f)$ with zeros for values of $m>M-1$ and using the FFT: 


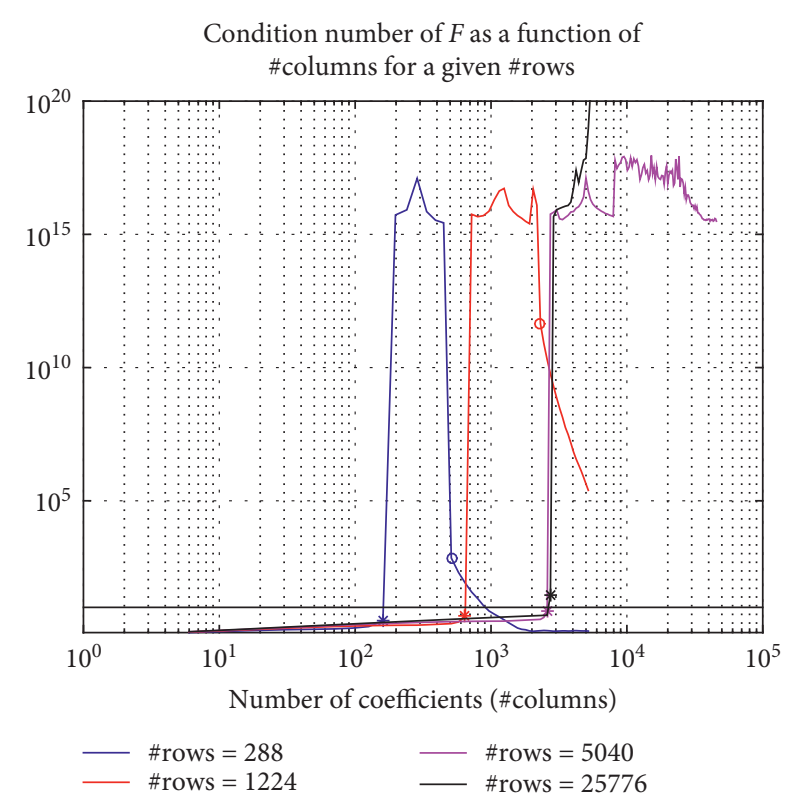

FIgURE 3: The condition number of the matrix $\mathbf{F}$ as a function of the number of columns. The horizontal black line denotes a condition number of 10 .

TABLE 4: Values of $Q_{n}(f)$ stored in bins.

\begin{tabular}{lcc}
\hline Bin index & Frequency & Stored value \\
\hline 0 & 0 & 0 \\
1 & $\Delta f$ & 0 \\
$\vdots$ & $\vdots$ & $\vdots$ \\
$N_{1}-1$ & $\left(N_{1}-1\right) \Delta f$ & 0 \\
$N_{1}$ & $N_{1} \Delta f$ & $Q_{n}\left(N_{1} \Delta f\right)$ \\
$\vdots$ & $\vdots$ & $\vdots$ \\
$N_{2}$ & $N_{2} \Delta f$ & $Q_{n}\left(N_{2} \Delta f\right)$ \\
$N_{2}+1$ & $\left(N_{2}+1\right) \Delta f$ & 0 \\
$\vdots$ & $\vdots$ & $\vdots$ \\
$(M / 2)-1$ & $((M / 2)-1) \Delta f$ & 0 \\
$(M / 2)$ & $-(M / 2) \Delta f$ & 0 \\
$\vdots$ & $\vdots$ & $\vdots$ \\
$M-N_{2}-1$ & $-\left(N_{2}+1\right) \Delta f$ & 0 \\
$M-N_{2}$ & $-N_{2} \Delta f$ & $Q_{n}^{*}\left(N_{2} \Delta f\right)$ \\
$\vdots$ & $\vdots$ & $\vdots$ \\
$M-N_{1}$ & $-N_{1} \Delta f$ & $Q_{n}^{*}\left(N_{1} \Delta f\right)$ \\
$M-N_{1}+1$ & $-\left(N_{1}-1\right) \Delta$ & 0 \\
$\vdots$ & $\vdots$ & $\vdots$ \\
$M-1$ & $-\Delta f$ & 0 \\
\hline
\end{tabular}

$$
Q_{n}\left(k \Delta f^{\prime}\right)=\sum_{m=0}^{M^{\prime}-1} q_{n}(m \Delta t) e^{-j\left(2 \pi k m / M^{\prime}\right)},
$$

where $M^{\prime}$ is the new number of bins and $\Delta f^{\prime}$ is the new frequency step which is equal to $\Delta f^{\prime}=1 /\left(M^{\prime} \Delta t\right)$. By choosing $M^{\prime}>M$ the frequency step, $\Delta f^{\prime}$ will be smaller than the original frequency step $\Delta f \cdot q_{n}(m \Delta t)=0$ for values of $m$ from $M$ to $M^{\prime}-1$.

So, in summary, the following procedure can be used to determine the values of $M$ and $M^{\prime}$ :

(i) $M$ should be larger than $2 N_{2}+2$ and a power of 2 . This determines a minimum value of $M$. (ii) $\Delta f$ should be chosen small enough such that the time domain spherical wave coefficients $q_{n}(m \Delta t)$ decay to zero within the time window $T=(1 / \Delta f)$.

(iii) If a finer resolution in the time domain is needed, the value of $M$ can be increased further $(\Delta t=1 /(M \Delta f)$.

(iv) The value of $M^{\prime}$ is determined by the required frequency step after interpolation: $M^{\prime}=1 /\left(\Delta f^{\prime} \Delta t\right)=\left(M \Delta f / \Delta f^{\prime}\right)$.

\section{Test Case: The LOFAR System}

3.1. General Description of the LOFAR Telescope. LOFAR (LOw Frequency ARray) is a radio telescope for radio astronomical observations in the frequency range of $10 \mathrm{MHz}$ to $240 \mathrm{MHz}$. It consists of 52 stations distributed over Europe. There are three kinds of stations: core stations, remote stations, and international stations. The core stations and remote stations are located in the Netherlands. The international stations are located in France, Germany, Ireland, Latvia, Poland, Sweden, and the United Kingdom. Each station consists of two antenna types: low-band antennas (LBA) and high-band antennas (HBA). The low-band antennas are designed for the frequency range of 10-90 MHz. The high-band antennas are designed for the frequency range of 110-240 MHz. Each low-band antenna consists of two orthogonal inverted- $\mathrm{V}$ dipoles. The highband antennas consist of two orthogonal bowtie antennas. A group of 16 high-band antennas is combined in a unit called a tile. The number of LBA and HBA tiles for the different kinds of stations is shown in Table 5. More information about LOFAR and its science cases can be found in [9].

Each LOFAR station is rotated by a particular angle such that the side lobes of each station beam point towards a different point in the sky. This minimizes the effect of the side lobes on the sky image because the effect of the side lobes is averaged [11]. The orientation of the individual antennas with respect to a common coordinate system for all stations is the same. The result is that the configuration of each individual station is unique.

3.2. Detailed Description of the Low-Band Antenna. The electromagnetic simulations are performed using FEKO [12]. The simulation model of a low-band antenna is shown in Figure 1.

Each low-band antenna consists of two inverted-V dipoles above a metal ground plane of $3 \times 3 \mathrm{~m}^{2}$. In this paper, we will use the term "element" to denote one of these dipoles. The term "dual-pol element" will be used to denote a group of two elements as shown in Figure 1. Each arm of the two inverted-V dipoles has a length of 1.38 meters and is excited separately. The height of the feed point above-ground plane is $1.7 \mathrm{~m}$. The dipole in the $\varphi=45^{\circ}$ plane is referred to as polarization 1 . The dipole in the $\varphi=135^{\circ}$ plane is referred to as polarization 2 . The outer conductor of the coaxial cable from the low-noise amplifier (LNA, positioned at the terminals marked by $V_{1, a}^{\mathrm{rec}}, V_{1, b}^{\mathrm{rec}}, V_{2, a}^{\mathrm{rec}}$, and $V_{2, b}^{\mathrm{rec}}$ in Figure 1) to the metal ground plane is modeled by a vertical wire with a 
TABLE 5: Number of LBA and HBA tiles for the different kinds of stations.

\begin{tabular}{lcr}
\hline Kind of station & Number of LBA & Number of HBA tiles \\
\hline Core & 96 & 48 (2 clusters of 24 tiles) \\
Remote & 96 & 48 (1 cluster of 48 tiles) \\
International & 96 & 96 (1 cluster of 96 tiles) \\
\hline
\end{tabular}

length of 1.7 meters. The coupling between the outer conductor and the metal ground plane is modeled as a perfect connection. In practice, this is a capacitive coupling. The metal plate is modeled as an infinite thin perfect conducting sheet. The wires are modeled as perfect conducting wires with a radius of $0.1 \mathrm{~mm}$. The metal ground plane is located at $z=0 \mathrm{~mm}$. The space at $z<0 \mathrm{~mm}$ is modeled as a dielectric with a relative dielectric constant $\varepsilon_{r}$ of 3 and a conductivity $\sigma$ of $0.01 \mathrm{~S} / \mathrm{m}$. This dielectric represents the soil. The space above $z=0 \mathrm{~mm}$ is modeled as a vacuum.

Each arm is excited by a voltage source. This means that each arm has its own embedded element pattern which is calculated by exciting its corresponding arm with $1 \mathrm{~V}$ while the other arms are short-circuited. These embedded element patterns are transformed to voltages over the single-ended LNAs in receive situation for the case the antenna is excited with a plane wave with an electrical field strength of $1 \mathrm{~V} / \mathrm{m}$. This transformation is described in the appendix and follows an approach similar to [13]. Exciting each dipole arm separately instead of each dipole gives the possibility to investigate the common mode behaviour of the antenna. This is however not discussed in this paper.

If the single-ended voltages across each single-ended LNA in receive situation are equal to the voltages $V_{1, a}^{\text {rec }}, V_{1, b}^{\text {rec }}$, $V_{2, a}^{\text {rec }}$, and $V_{2, b}^{\text {rec }}$ as shown in Figure 1 , the open-circuit differential voltages of polarizations 1 and 2 can be written as

$$
\begin{aligned}
& V_{\mathrm{diff}, 1}^{\mathrm{rec}}=V_{1, a}^{\mathrm{rec}}-V_{1, b}^{\mathrm{rec}}, \\
& V_{\mathrm{diff}, 2}^{\mathrm{rec}}=V_{2, a}^{\mathrm{rec}}-V_{2, b}^{\mathrm{rec}},
\end{aligned}
$$

where $V_{\text {diff, } n}^{\text {rec }}$ with $n=1,2$ being the differential voltage for polarization $n$. Note that the load impedance used to calculate the single-ended voltages should be equal to the single-ended LNA impedance (half the differential mode impedance).

3.3. Description of Embedded Element Patterns. In this section, we will discuss the amount of data that is needed to store all embedded element patterns of a LOFAR station and the possibility of reducing this. The dataset of the embedded element patterns of each dual-pol element consists of four complex numbers per direction per frequency point: a $\theta$ - and $\varphi$-component for each element (each dual-pol element consists of two orthogonal elements).

For this paper, we simulated the embedded element patterns from $\theta=0^{\circ}$ to $\theta=90^{\circ}$ in steps of $1^{\circ}$ and from $\varphi=0^{\circ}$ to $\varphi=355^{\circ}$ in steps of $5^{\circ}$. Table 6 shows the amount of complex numbers that is needed in this case for the different kind of LOFAR stations.

In general, the element patterns are not independent. This means that an element pattern of an arbitrary element can be written as a linear combination of the element patterns of other elements. If we can write the element patterns as a sum of orthogonal element patterns, the amount of data can be reduced because the number of orthogonal element patterns is less than the number of element patterns. To describe the element patterns only the coefficients of the linear combination have to be stored besides the orthogonal element patterns. The element patterns of an array of $N_{\text {elem }}$ elements can be written as a $2 N_{\text {dir }} \times N_{\text {elem }}$ matrix E:

$$
\mathbf{E}=\left[\mathbf{e}_{1} \ldots \mathbf{e}_{N_{\text {elem }}}\right] \text {, }
$$

where $\mathbf{e}_{i}$ is the element pattern as defined in equation (11) of element $i$. Each column of $\mathbf{E}$ represents an element pattern.

Using a singular value decomposition, the matrix $\mathbf{E}$ can be written as

$$
\mathbf{E}=\mathbf{U} \mathbf{D W}^{H},
$$

where $\mathbf{D}$ is a $2 N_{\text {dir }} \times N_{\text {elem }}$ diagonal matrix containing the singular values sorted from large to small, $\mathbf{U}$ and $\mathbf{W}$ are unitary matrices of size $2 N_{\text {dir }} \times 2 N_{\text {dir }}$ and $N_{\text {elem }} \times N_{\text {elem }}$, respectively, and $H$ denotes the Hermitian operator. The matrix $\mathbf{E}$ can be approximated by

$$
\mathbf{E} \approx \mathbf{U}_{\text {basis }} \mathbf{D}_{\text {basis }} \mathbf{W}_{\text {basis }}^{H}
$$

where $\mathbf{D}_{\text {basis }}$ is a $N_{\text {basis }} \times N_{\text {basis }}$ diagonal matrix containing the $N_{\text {basis }}$ largest singular values. It is assumed that smaller singular values can be neglected. The matrices $\mathbf{U}_{\text {basis }}$ and $\mathbf{W}_{\text {basis }}$ are constructed by taking the first $N_{\text {basis }}$ columns of $\mathbf{U}$ and $\mathbf{W}$ and therefore have sizes $2 N_{\text {elem }} \times N_{\text {basis }}$ and $N_{\text {basis }} \times N_{\text {basis }}$, respectively. The columns of $\mathbf{U}_{\text {basis }}$ are a set of orthonormal basis functions for the element patterns.

Figure 4 shows the number of dimensions $N_{\text {basis }}$ for the LOFAR low-band array as a function of frequency for frequencies from 10 to $90 \mathrm{MHz}$ in steps of $5 \mathrm{MHz}$ (17 frequencies) and $1 \mathrm{MHz}$ (81 frequencies). It is assumed that singular values that are less than 0.01 times the largest singular value can be neglected.

Without using the singular value decomposition, one would need $2 \times 96 \times 17=3264$ embedded element patterns for 17 frequencies and $2 \times 96 \times 81=15552$ embedded element patterns for 81 frequencies. If singular value decomposition is used, only 543 (the sum of the number of dominant dimensions $N_{b}$ for each frequency) element patterns are needed as a basis function for 17 frequency points. This means that only $543 / 3264=16.6 \%$ of the amount of data is needed to describe the element patterns for 17 frequency points. For 81 frequency points, these numbers are 2610 dimensions and $2610 / 15552=16.8 \%$, respectively. This is a reduction of the number of embedded element 
TABle 6: Number of complex numbers needed to describe element patterns of one station.

\begin{tabular}{lccc}
\hline Kind of station & Antenna type & Number of dual-pol elements & Number of complex numbers per frequency point \\
\hline Core and remote & LBA & 96 & 2515968 \\
Core and remote & HBA & 768 & 20127744 \\
International & LBA & 96 & 2515968 \\
International & HBA & 1536 & 40255488 \\
\hline
\end{tabular}

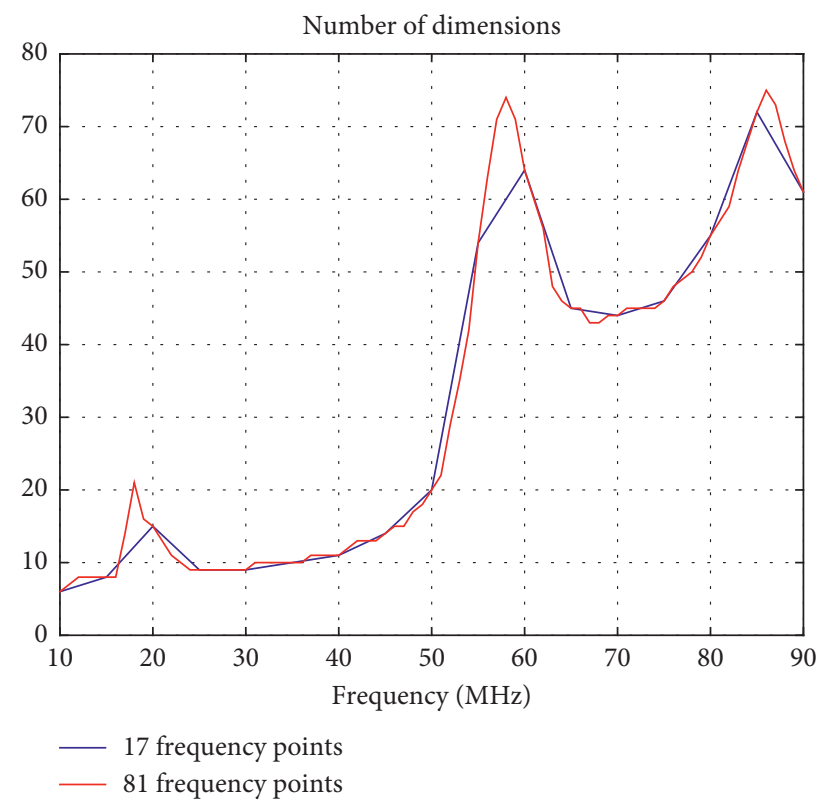

Figure 4: Number of dominant dimensions of the embedded element patterns of the LOFAR low-band array as a function of frequency.

patterns that is needed to describe the low-band array. The following section demonstrates that the technique using spherical wave expansion, proposed in this work, reduces the data needed to describe each embedded element pattern.

\section{Results}

4.1. Verification. As a test case, the embedded element patterns of one of the core stations (CS302) are described using spherical wave expansion. The layout of the simulated station is shown in Figure 5. Element 1 is the center element. Elements 46 and 47 are the outer elements. The distance between these elements and the other ones is more than 20 meters.

To apply spherical wave expansion, one has to determine $N_{\max }$ in equation (9). In theory, the radius $r_{0}$ should be chosen such that the whole station will fit in a sphere with that radius. That would mean that $r_{0}$ should be equal to around $65 \mathrm{~m}$. In practice, the embedded element pattern of an element is only determined by its closest neighbours. This means that $r_{0}$ can have a lower value than $65 \mathrm{~m}$. For a given $N_{\max }, r_{0}$ is equal to

$$
r_{0}=\frac{N_{\max }-10}{\beta} .
$$

It turned out that the coupling between an element for which the embedded element pattern is calculated and the elements further away than $r_{0}$ is less than $-35 \mathrm{~dB}$ for all simulated frequencies if $N_{\max }=31$. This value is used for the calculations in this paper.

According to equation (8), $N_{\max }=31$ corresponds with 2046 spherical wave coefficients. The patterns are calculated for the same $\theta$ - and $\varphi$-angles as in Section 3.3. The matrix $\mathbf{F}$ defined in (13) is calculated for $\theta=1^{\circ}$ to $\theta=179^{\circ}$ in steps of $1^{1^{\circ}}$ and from $\varphi=0^{\circ}$ to $\varphi=355^{\circ}$ in steps of $5^{\circ}$. The normalized electric field $\mathbf{e}$ is set to zero for $\theta>90^{\circ}$. The resulting size of $\mathbf{F}$ is $25776 \times 2046$. Its condition number is 4.8 , which shows that the sensitivity of the solution of (16) for deviations in the vector $\mathbf{e}$ is small. The rank of $\mathbf{F}$ is 2046 which means that the matrix has a full column rank. So, there is one solution for which the norm $\|\mathbf{e}-\mathbf{F q}\|_{2}$ has its minimum.

Figures 6 and 7 show the simulated embedded element pattern $\left(V_{\text {sim }}\right)$ of element 47 (one of the two "isolated: elements) in the $\varphi=45^{\circ}$ plane at $57 \mathrm{MHz}$ together with the same embedded element pattern reconstructed from the calculated spherical mode coefficients $\left(V_{\text {recon }}\right)$ and the difference between the simulated and reconstructed patterns (EES). The difference in amplitude is expressed as an Equivalent Error Signal (EES). The EES expressed in $\mathrm{dB}$ is defined as

$$
\mathrm{EES}=20 \log _{10}|| V_{\text {recon }}|-| V_{\text {sim }} \| .
$$

The frequency of $57 \mathrm{MHz}$ is chosen because this is the resonance frequency of the inverted- $\mathrm{V}$ antenna used in the LBA. The embedded element patterns are plotted as the voltage at the input of the LNA for the case of an incoming plane wave with a field strength of $1 \mathrm{~V} / \mathrm{m}$.

One can see that the EES is less than $-30 \mathrm{~dB}$ and the phase difference is less than $0.5^{\circ}$ in phase for most angles.

The simulated and reconstructed embedded element patterns for element 1 in the $\varphi=45^{\circ}$ plane at $57 \mathrm{MHz}$ are shown in Figures 8 and 9. This element is the center element of the LBA station, within the densest part of the array. Due to the mutual coupling, the embedded element patterns have an irregular shape in this part of the array, especially around the resonance frequency of $57 \mathrm{MHz}$. So, the embedded element pattern of the element at $57 \mathrm{MHz}$ can be considered as the worst case for the description of the embedded element patterns by spherical wave expansion.

It is clearly visible that the element pattern has a minimum around $\theta=20^{\circ}$. This minimum is caused by the mutual coupling between the antennas and is also validated by a measurement [14]. The EES is less than $-20 \mathrm{~dB}$. The phase difference is $7^{\circ}$ around the amplitude dip at for $\theta=20^{\circ}$ and less than $2^{\circ}$ for other angles.

The embedded element patterns are described by 2046 coefficients (i.e., complex numbers) per simulated frequency. 


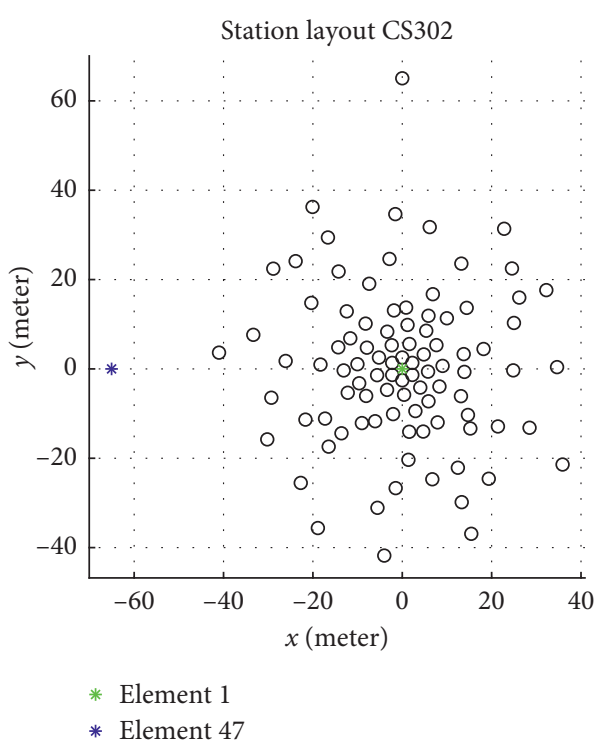

FIgURE 5: Layout of the low-band array of the simulated LOFAR station (CS302).

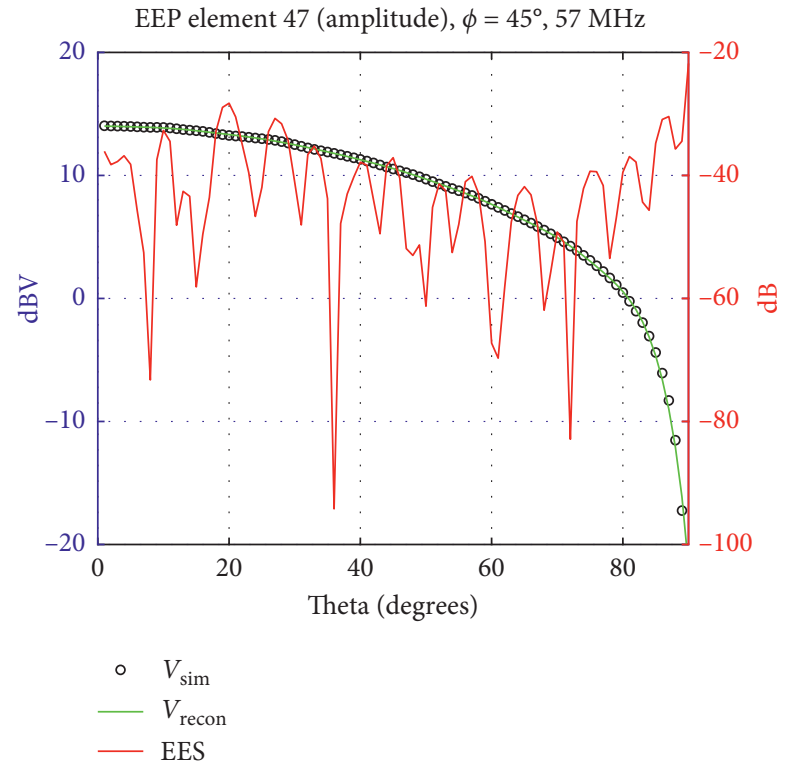

FIGURE 6: Simulated and reconstructed EEP of element 47 (amplitude).

Comparing this number with the amount of numbers needed to describe the embedded element patterns directly (in our case $90 \theta$-angles $\times 72 \varphi$-angles $\times 2$ field components $=12960)$ does not make sense because this number can be chosen arbitrarily. A better number to compare with is the minimum amount of complex numbers needed to describe the embedded element patterns to fulfill the Nyquist criterion in the spatial domain. To determine this number, we interpolated the embedded element patterns to get data points on a regular $u$, $v$-grid. These data points were transformed to the spatial frequency domain using a discrete Fourier transform. For the embedded element pattern of the center element at $57 \mathrm{MHz}$, it turned out that 2449

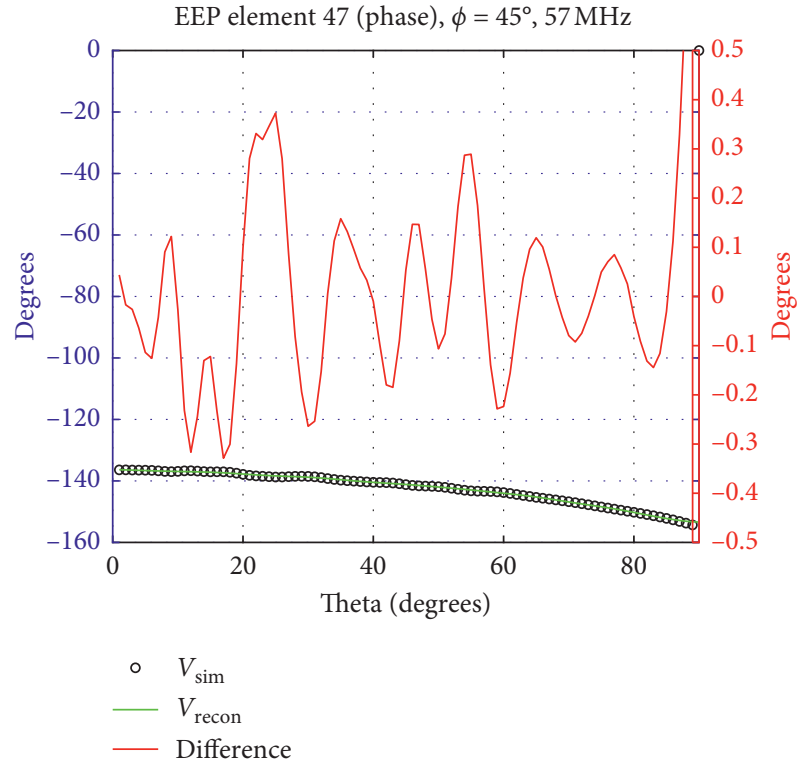

FIgURE 7: Simulated and reconstructed EEP of element 47 (phase).

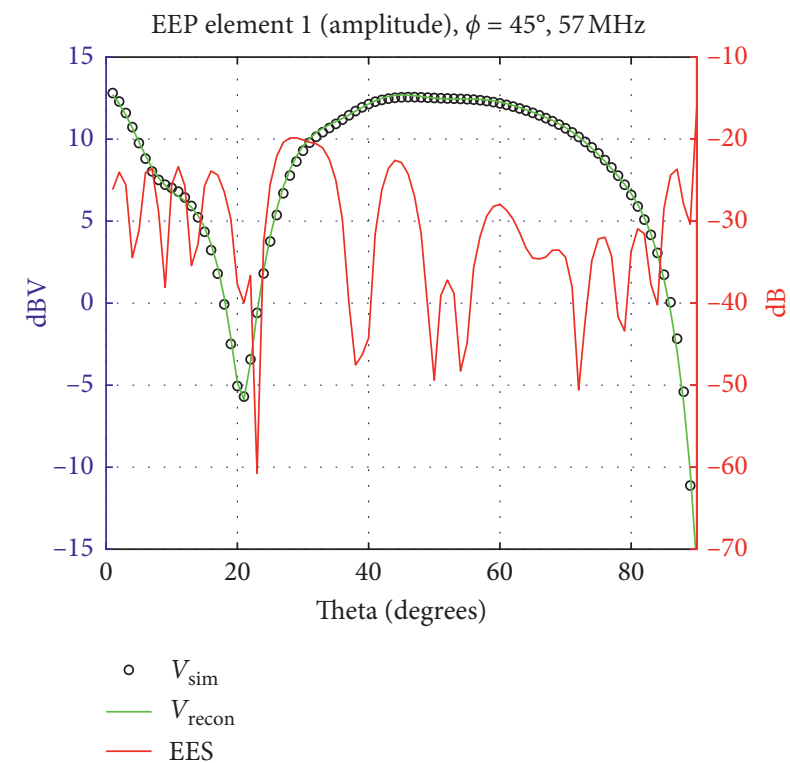

Figure 8: Simulated and reconstructed EEP of element 1 (amplitude).

sample points were needed to have $99.9 \%$ of the energy included. Because every sample point contains two complex numbers (one for each field component), this means that 4898 complex numbers are needed to describe each embedded element pattern. There were 2046 coefficients needed to describe the embedded element pattern. Because spherical wave expansion considers the total field (both field components), the amount of complex numbers to describe each embedded element pattern is the same. This means that by using spherical wave expansion $2046 / 4898=41.8 \%$ of the data is needed to describe the embedded element pattern at $57 \mathrm{MHz}$ compared to direct storage of it sampled at the Nyquist rate. 


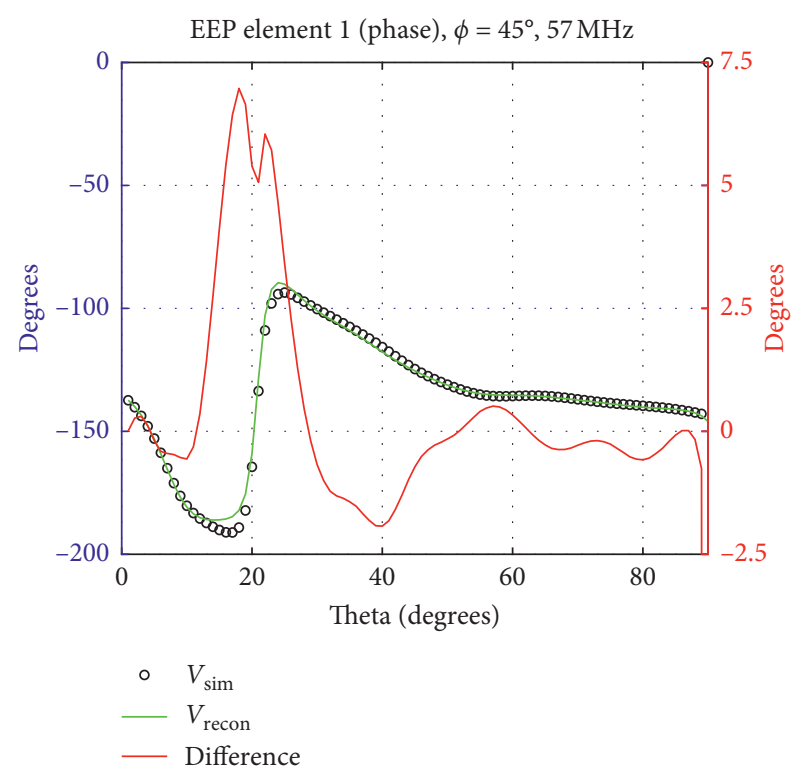

FIgURE 9: Simulated and reconstructed EEP of element 1 (phase).

4.2. Interpolation. The LBA is simulated for frequencies between 10 and $90 \mathrm{MHz}$ in discrete steps. In this subsection, we will show the application of the interpolation method described in Section 2.3.

Figure 10 shows the time domain response (impulse response) of the electric field at $10 \mathrm{~m}$ distance at $\theta=1^{\circ}$ and $\varphi=45^{\circ}$ if 33 frequency points between 10 and $90 \mathrm{MHz}$ are used. This corresponds with a frequency resolution $\Delta f$ of $2.5 \mathrm{MHz}$ and a period $T$ of $400 \mathrm{~ns}$ in the time domain. The size $M$ of the used inverse FFT is 1024 points which corresponds with a time domain step of $\Delta t=1 /(M \Delta f)=$ $1 /\left(1024 \cdot 2.5 \cdot 10^{6}\right) \mathrm{Hz}=0.39 \mathrm{~ns}$. It can be seen that the time domain response does not decay to zero. This means that the frequency step is too coarse.

The time domain response for the case that the frequency step $\Delta f$ is decreased to $1 \mathrm{MHz}$ ( 81 frequency points) is shown in Figure 11. The corresponding length of the period $T$ in the time domain is $1000 \mathrm{~ns}$.

One can see that the time domain response decays to almost zero (less than $1 \%$ of the maximum amplitude). This means that a frequency resolution of $1 \mathrm{MHz}$ is sufficient to interpolate the spherical wave coefficients. To illustrate the interpolation method, the frequency step is reduced to $\Delta f^{\prime}=0.5 \mathrm{MHz}$. This means that the number of bins in the FFT has to be increased to $M^{\prime}=\left(M \Delta f / \Delta f^{\prime}\right)=(1024$. $\left.1 \cdot 10^{6} / 0.5 \cdot 10^{6}\right)=2048$. The spherical wave coefficients in the time domain are padded with 1024 zeros.

Figures 12 and 13 show the amplitude and phase of the embedded element pattern at $57.5 \mathrm{MHz}$ in the $\varphi=45^{\circ}$ plane. $V_{\text {sim }}$ is the simulated pattern, $V_{\text {recon }}$ is the embedded element pattern obtained from the calculated spherical wave coefficients, and $V_{\text {interp,fft }}$ is the embedded element pattern obtained from the spherical wave coefficients obtained by FFT-interpolation. As a reference, the amplitude and phase of the embedded element patterns obtained by linear interpolation $\left(V_{\text {interp,linear }}\right)$ and cubic spline interpolation

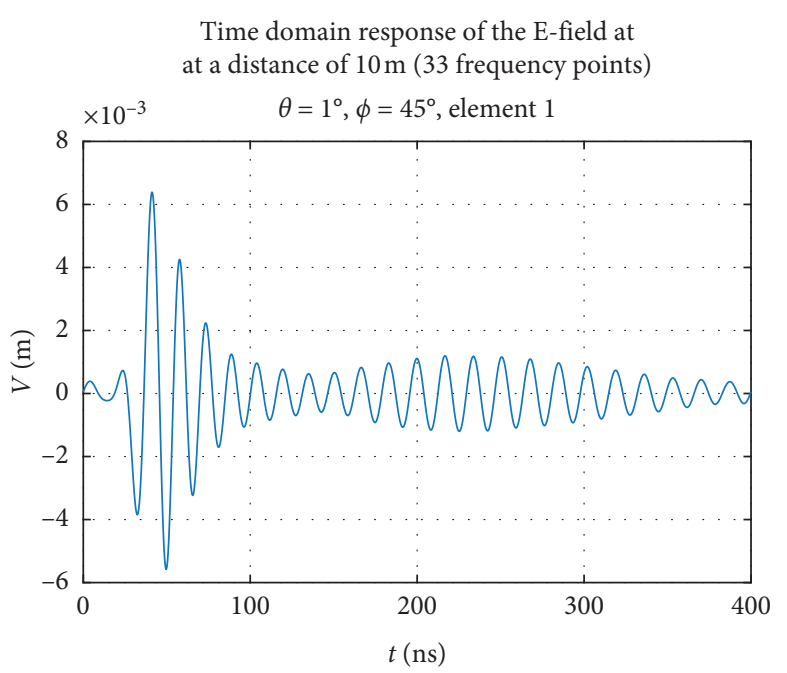

FIgURE 10: Impulse response of the E-field using 33 frequency points.

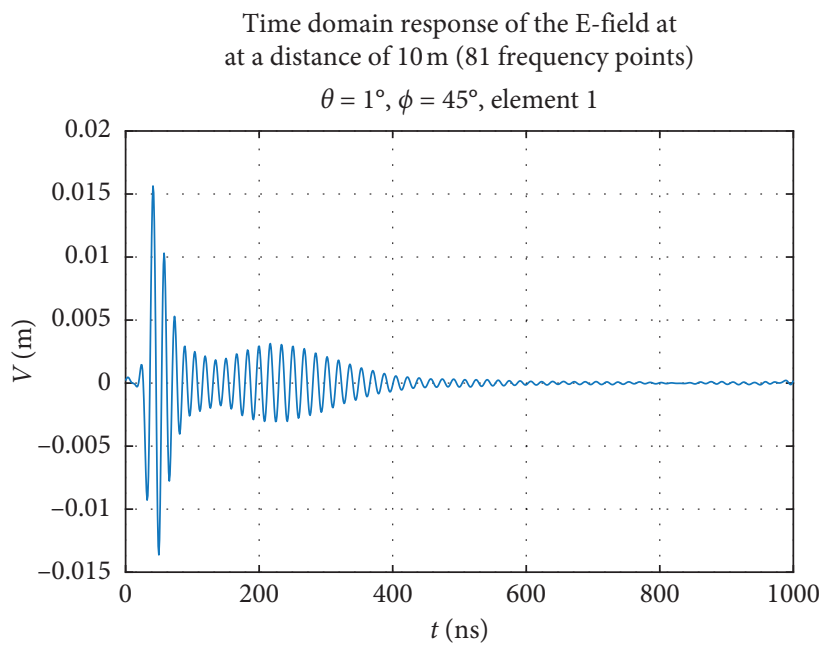

FIGURE 11: Impulse response of the E-field using 81 frequency points.

( $\left.V_{\text {interp,spline }}\right)$ of the spherical wave coefficients are also included.

Figure 14 shows several Equivalent Error Signals (EES). EES $_{\alpha}$ is the EES based on the difference between $V_{\beta}$ and $V_{\alpha}$, where $\beta$ stands for recon, interp, fft, interp, linear, or interp, spline and $\alpha$ stands for sim or recon.

Figure 15 shows the phase differences corresponding to the Equivalent Error Signals of Figure 14.

One can see that the differences between the interpolated and reconstructed embedded element patterns, on one hand, and the simulated embedded element pattern, on the other hand, are comparable. The EEP has a maximum of $-18 \mathrm{~dB}$ and the difference in phase has a maximum of $5^{\circ}$ except around $\theta=20^{\circ}$. The difference between the interpolated and reconstructed embedded element pattern is less (EEP less than $-30 \mathrm{~dB}$, phase difference less than $1^{\circ}$ (except around $\left.\theta=20^{\circ}\right)$ ). This means that the error due to the interpolation is less than the error due to the use of spherical wave 


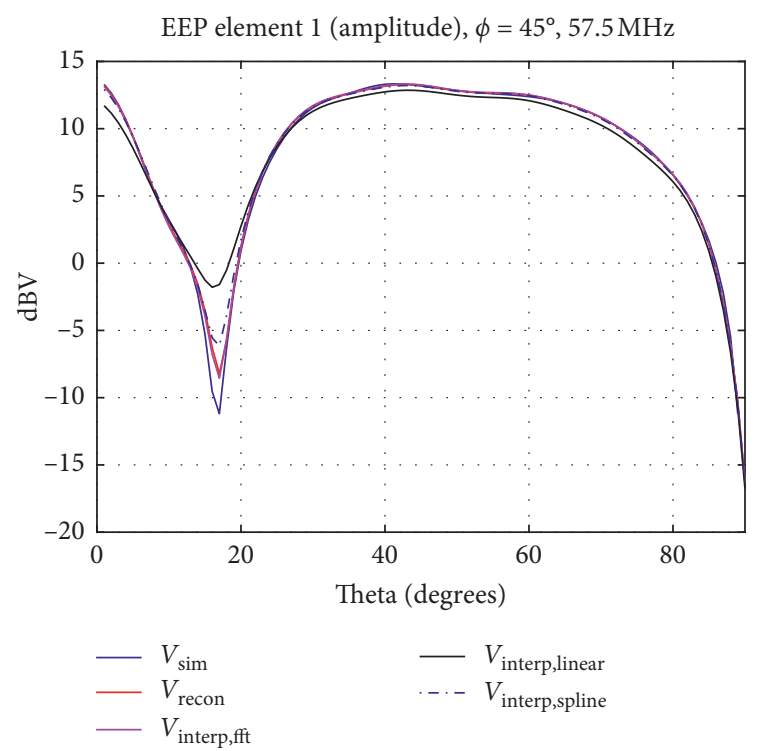

Figure 12: Simulated, reconstructed, and interpolated EEP of element 1 at $57.5 \mathrm{MHz}$ (amplitude).

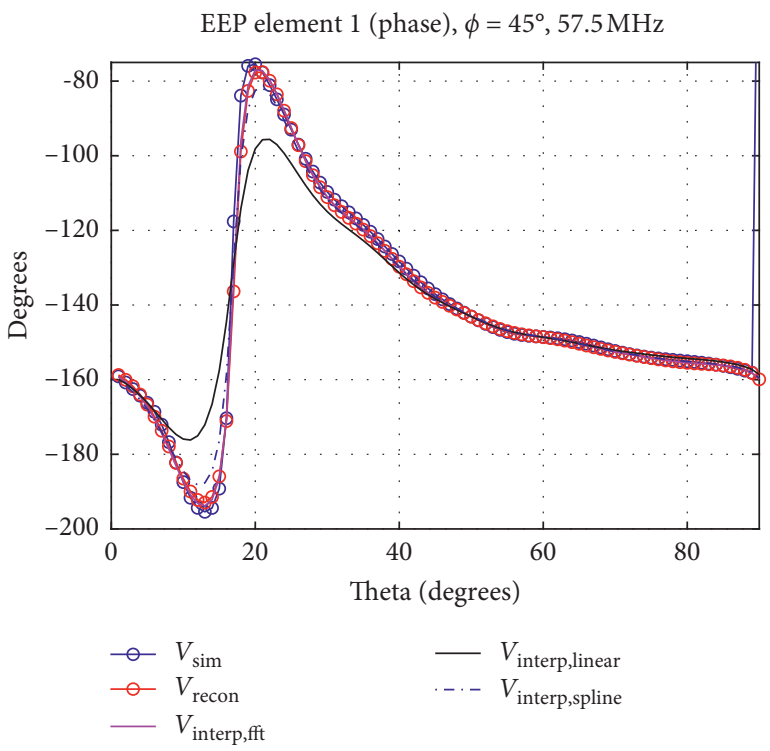

Figure 13: Simulated, reconstructed, and interpolated EEP of element 1 at $57.5 \mathrm{MHz}$ (phase).

expansion and it shows that the error due to the interpolation is not significant. So, using the FFT for interpolation eliminates the need to perform a full-wave electromagnetic simulation of the embedded element patterns with a frequency resolution of less than $1 \mathrm{MHz}$. From Figures 14 and 15 , it is also clear that using the FFT for interpolation of the spherical wave coefficients gives a lower EES and phase difference compared to linear interpolation and cubic spline interpolation.

If the embedded element patterns between 10 and $90 \mathrm{MHz}$ would be simulated with a frequency resolution of $0.5 \mathrm{MHz}$, a full-wave electromagnetic simulation of 161 frequency points would be needed. By using FFT-

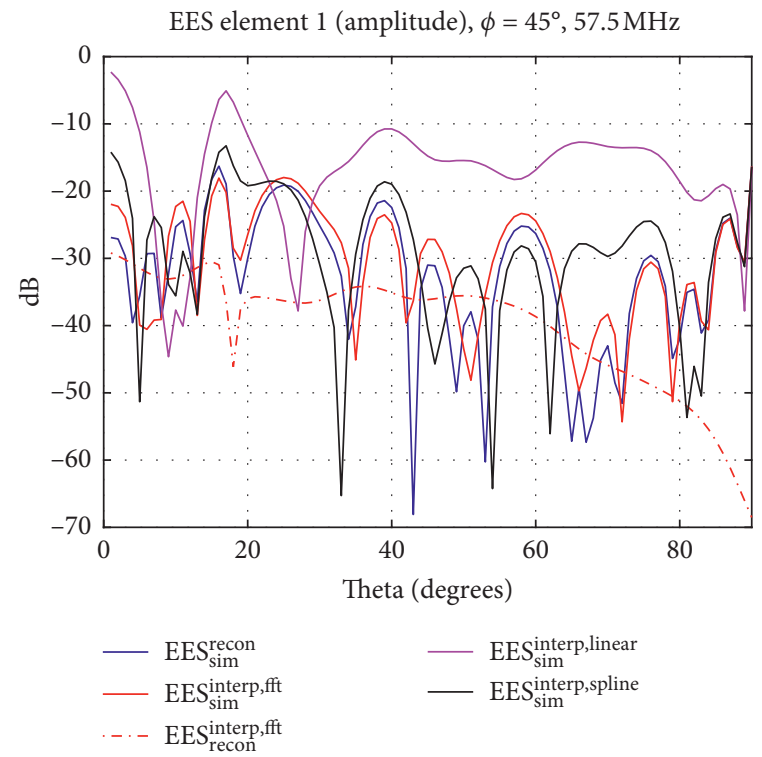

FIgUre 14: EES of the EEP of element 1 at $57.5 \mathrm{MHz}$ (amplitude).

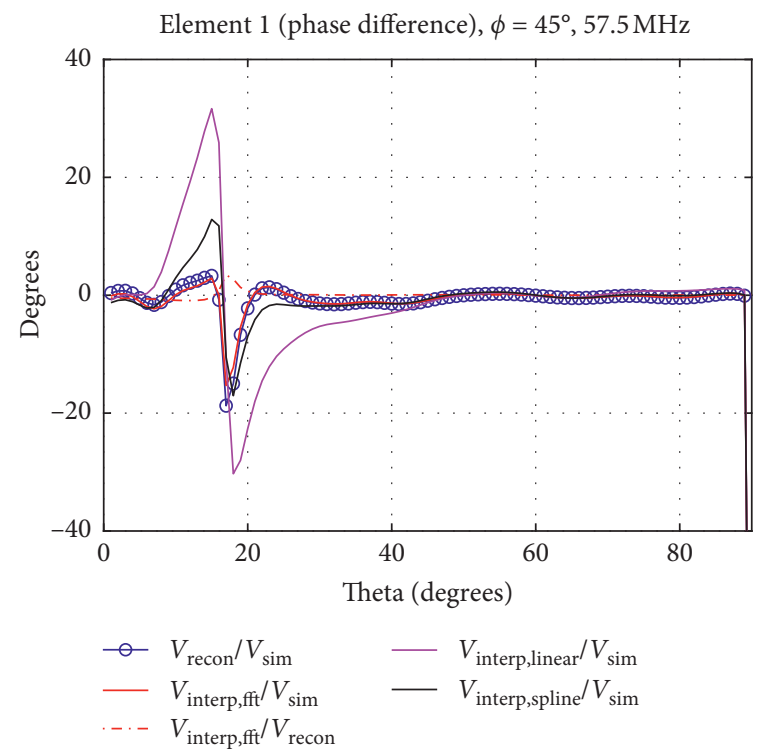

FIgURE 15: Phase differences of EEP of element 1 at $57.5 \mathrm{MHz}$ (phase).

interpolation only 81 frequency points have to be simulated, which is a reduction of 80 frequency points. The cost for this reduction is to perform FFTs, which can be performed in less than 1 minute on a modern laptop computer. This is considerably less than a full-wave electromagnetic simulation of a complete LBA station at 80 additional frequency points on a dedicated server (which takes approximately 7 hours per frequency point).

\section{Conclusions}

This paper shows that it is possible to use spherical wave expansion to describe the embedded element patterns of the LOFAR low-band array by the coefficients of spherical wave 
functions. The example showed that $41.8 \%$ of the data is needed to describe the embedded element patterns, compared to direct storage of the embedded element patterns sampled at the Nyquist rate. It turned out that the original embedded element patterns could be reconstructed with an EES of less than $-20 \mathrm{~dB}$ and a phase error better than $2^{\circ}$. Only at the dip in the pattern, the accuracy is less.

By using an FFT, it is possible to interpolate the embedded element patterns in the frequency domain. It turned out that a frequency resolution of $1 \mathrm{MHz}$ is sufficient to interpolate the embedded element patterns. The main difference with respect to the simulated pattern is caused by the use of spherical wave expansion. It is also shown that the error due to interpolation by using the FFT is lower compared to the use of linear interpolation or cubic spline interpolation.

\section{Appendix}

\section{Transformation of the Embedded Element Patterns in Transmit Situation to Embedded Element Patterns in Receive Situation for a Given Load Impedance}

The embedded element patterns of an array in receive situation depend on the load impedance terminating the array elements. The simulation software used for the calculation of the low-band array calculates the Z-matrix and the embedded element patterns in transmit situation using voltage sources. This means that, for the calculation of each embedded element pattern, one element is excited with a voltage source of $1 \mathrm{~V}$ while the other elements are shortcircuited. These embedded element patterns and the impedance matrix $\mathbf{Z}$ of the antenna will be used to calculate the voltages across the load impedances in receive situation. The advantage of this approach is that these voltages can be calculated in postprocessing, so the embedded element patterns for different load impedances can be calculated without rerunning the electromagnetic simulation. This appendix describes this postprocessing procedure.

The embedded element patterns in transmit situation for the case that the array is excited with voltage sources can be written as an $N_{\text {ports }} \times 2$ matrix $\mathbf{p}_{\mathbf{y}}\left(\widehat{u}_{r}\right)$ where $N_{\text {ports }}$ is the number of elements:

$$
\mathbf{p}_{\mathbf{y}}\left(\widehat{u}_{r}\right)=\left[\begin{array}{cc}
e_{1, \theta}^{y}\left(\widehat{u}_{r}\right) & e_{1, \varphi}^{y}\left(\widehat{u}_{r}\right) \\
\vdots & \vdots \\
e_{N_{\text {ports }}, \theta}^{y}\left(\widehat{u}_{r}\right) & e_{N_{\text {ports }}, \varphi}^{y}\left(\widehat{u}_{r}\right)
\end{array}\right],
$$

where $e_{i, \theta}^{y}\left(\widehat{u}_{r}\right)$ and $e_{i, \varphi}^{y}\left(\widehat{u}_{r}\right)$ are $\theta$ - and $\varphi$-component of the normalized electric field in the direction $\widehat{u}_{r}$ as defined in (1) for the case element $i$ is excited while the other ports are shorted. The total field $\mathbf{p}_{\text {tot }}$ of the array can be written as

$$
\mathbf{p}_{\text {tot }}\left(\widehat{u}_{r}\right)=\left(\mathbf{V}^{\text {trans }}\right)^{T} \mathbf{p}_{\mathbf{y}}\left(\widehat{u}_{r}\right) \text {, }
$$

where the vector $\mathbf{V}^{\text {ports }}$ is an $N_{\text {ports }}$-elements column vector containing the excitation voltages of each element. The row vector $\mathbf{p}_{\text {tot }}\left(\widehat{u}_{r}\right)$ contains the normalized electric field in a direction $\widehat{u}_{r}$ :

$$
\mathbf{p}_{\text {tot }}\left(\widehat{u}_{r}\right)=\left[e_{\text {tot }, \theta}\left(\widehat{u}_{r}\right) e_{\text {tot }, \varphi}\left(\widehat{u}_{r}\right)\right] .
$$

In a similar way, the embedded element patterns for the case the elements are excited with current sources (while the nonexcited elements are open) can be written in a matrix $\mathbf{p}_{\mathbf{z}}\left(\widehat{u}_{r}\right)$. The total electric field $\mathbf{p}_{\text {tot }}\left(\widehat{u}_{r}\right)$ can now be written as

$$
\mathbf{p}_{\text {tot }}\left(\widehat{u}_{r}\right)=\left(\mathbf{I}^{\text {trans }}\right)^{T} \mathbf{p}_{\mathbf{z}}\left(\widehat{u}_{r}\right) \text {. }
$$

The column vector $\mathbf{I}^{\text {trans }}$ contains the excitation currents of each element. By equating (A.2) and (A.4) and substituting $\mathbf{V}^{\text {trans }}=\mathbf{Z} \mathbf{I}^{\text {trans }}, \mathbf{p}_{\mathbf{z}}\left(\widehat{u}_{r}\right)$ can be written as

$$
\mathbf{p}_{\mathbf{z}}\left(\widehat{u}_{r}\right)=\mathbf{Z}^{T} \mathbf{p}_{\mathbf{y}}\left(\widehat{u}_{r}\right)=\mathbf{Z} \mathbf{p}_{\mathbf{y}}\left(\widehat{u}_{r}\right)
$$

Because, for a reciprocal Z-matrix, $\mathbf{Z}=\mathbf{Z}^{T}$.

The open-circuit voltage $V_{i}^{\text {open }}\left(\widehat{u}_{r}\right)$ at port $i$ of an array antenna is given by

$$
V_{i}^{\text {open }}\left(\widehat{u}_{r}\right)=\vec{g}_{\mathrm{z}, i}\left(\widehat{u}_{r}\right) \cdot \vec{A}\left(\widehat{u}_{r}\right),
$$

where $\vec{A}\left(\widehat{u}_{r}\right)$ is the (complex) amplitude of an incident plane wave with an electric field $\vec{E}^{\text {nnt }}\left(\vec{r}, \widehat{u}_{r}\right)$ at position $\vec{r}$ coming from direction $\widehat{u}_{r}$ :

$$
\vec{E}^{\text {inc }}\left(\vec{r}, \widehat{u}_{r}\right)=\vec{A}\left(\widehat{u}_{r}\right) e^{j \beta \vec{r} \cdot \widehat{u}_{r}},
$$

and $\vec{g}_{z, i}\left(\widehat{u}_{r}\right)$ is the "voltage receive pattern" given by

$$
\vec{g}_{\mathrm{z}, i}\left(\widehat{u}_{r}\right)=g_{i, \theta}^{\mathrm{z}}\left(\widehat{u}_{r}\right) \widehat{u}_{\theta}+g_{i, \varphi}^{\mathrm{z}}\left(\widehat{u}_{r}\right) \widehat{u}_{\varphi} .
$$

The relation between voltages and currents at the ports of an array antenna can now be written as

$$
\mathbf{V}^{\mathrm{rec}}=\mathbf{Z} \mathbf{I}^{\mathrm{rec}}+\mathbf{g}_{\mathbf{z}}\left(\widehat{u}_{r}\right) \mathbf{A}\left(\widehat{u}_{r}\right)
$$

where $\mathbf{g}_{\mathbf{z}}\left(\widehat{u}_{r}\right)$ is an $N_{\text {ports }} \times 2$ matrix containing the voltage receive patterns of all array elements for a specific direction $\widehat{u}_{r}$ :

$$
\mathbf{g}_{\mathbf{z}}\left(\widehat{u}_{r}\right)=\left[\begin{array}{cc}
g_{1, \theta}^{z}\left(\widehat{u}_{r}\right) & g_{1, \varphi}^{z}\left(\widehat{u}_{r}\right) \\
\vdots & \vdots \\
g_{N_{\text {ports }}, \theta}^{z}\left(\widehat{u}_{r}\right) & g_{N_{\text {ports }}, \varphi}^{z}\left(\widehat{u}_{r}\right)
\end{array}\right] .
$$

The vector $\mathbf{A}\left(\widehat{u}_{r}\right)$ is a two-element column vector containing the complex amplitude of the $\theta$ - and $\varphi$-component of an incoming plane wave from direction $\widehat{u}_{r}$ :

$$
\mathbf{A}\left(\widehat{u}_{r}\right)=\left[\begin{array}{l}
A_{\theta}\left(\widehat{u}_{r}\right) \\
A_{\varphi}\left(\widehat{u}_{r}\right)
\end{array}\right],
$$

where $A_{\theta}\left(\widehat{u}_{r}\right)$ and $A_{\varphi}\left(\widehat{u}_{r}\right)$ are the $\theta$-and $\varphi$-components of $\vec{A}\left(\widehat{u}_{r}\right)$ :

$$
\vec{A}\left(\widehat{u}_{r}\right)=A_{\theta}\left(\widehat{u}_{r}\right) \widehat{u}_{\theta}+A_{\varphi}\left(\widehat{u}_{r}\right) \widehat{u}_{\varphi} .
$$


The relation between $\mathbf{p}_{\mathbf{z}}$ and $\mathbf{g}_{\mathbf{z}}$ is given by [15]

$$
\mathbf{g}_{\mathbf{z}}=\frac{4 \pi}{j \omega \mu_{0}} \mathbf{p}_{\mathbf{z}} \text {. }
$$

If the array in receive situation is described in terms of incoming and reflected waves $\mathbf{a}_{\text {rec }}$ and $\mathbf{b}_{\text {rec }}$, respectively, and a scattering matrix $\mathbf{S}$, the reflected wave can be written as

$$
\mathbf{b}_{\mathrm{rec}}=\mathbf{S} \mathbf{a}_{\mathrm{rec}}+\mathbf{g}_{\mathrm{s}} \mathbf{A} \text {, }
$$

where $\mathbf{g}_{\mathrm{s}}$ is an $N_{\text {ports }} \times 2$ matrix containing the "voltage wave receiving patterns" in a similar way to (A.10). The voltage wave receiving pattern is the reflected voltage wave for the case all the ports of the array are terminated with the reference impedance for which $\mathbf{a}_{\text {rec }}$ and $\mathbf{b}_{\text {rec }}$ are calculated (i.e., $\mathbf{a}_{\text {rec }}=0$ ). The relation between the voltages $\mathbf{V}_{\text {rec }}$ and $\mathbf{I}_{\text {rec }}$ and the voltage waves $\mathbf{a}_{\text {rec }}$ and $\mathbf{b}_{\text {rec }}$ is given by [16]

$$
\begin{aligned}
& \mathbf{a}_{\text {rec }}=\mathbf{F}\left(\mathbf{V}_{\text {rec }}+\mathbf{Z}_{0} \mathbf{I}_{\text {rec }}\right), \\
& \mathbf{b}_{\text {rec }}=\mathbf{F}\left(\mathbf{V}_{\text {rec }}-\mathbf{Z}_{0}^{*} \mathbf{I}_{\text {rec }}\right),
\end{aligned}
$$

where $\mathbf{Z}_{0}$ is a diagonal matrix containing the (complex) reference impedances for each antenna port. We will use the notation $\mathbf{Z}_{0}=\mathbf{R}_{0}+j \mathbf{X}_{0}$ where $\mathbf{R}_{0}$ is a diagonal matrix with the real part of the reference impedances and $\mathbf{X}_{0}$ is a diagonal matrix with the imaginary part of the reference impedances. In this paper, it is assumed that the real part of the reference impedance is positive. The matrix $\mathbf{F}$ in equations (A.15) and (A.16) is equal to

$$
\mathbf{F}=\frac{1}{2} \mathbf{R}_{0}^{-(1 / 2)}
$$

where the notation $\mathbf{R}_{0}^{-(1 / 2)}$ is used for a diagonal matrix containing the diagonal elements $\left(1 / \sqrt{R_{0, i}}\right)$ with $R_{0, i}$ the real part of the reference impedance of port $i$. The voltages $\mathbf{V}_{\text {rec }}$ and currents $\mathbf{I}_{\text {rec }}$ can be expressed as a function of the incident waves $\mathbf{a}_{\text {rec }}$ and reflected waves $\mathbf{b}_{\text {rec }}$ :

$$
\begin{aligned}
& \mathbf{V}_{\text {rec }}=\mathbf{F}^{-1}\left(\mathbf{Z}_{0}+\mathbf{Z}_{0}^{*}\right)^{-1}\left(\mathbf{Z}_{0}^{*} \mathbf{a}_{\text {rec }}+\mathbf{Z}_{0} \mathbf{b}_{\text {rec }}\right), \\
& \mathbf{I}_{\text {rec }}=\left(\mathbf{Z}_{0}+\mathbf{Z}_{0}^{*}\right)^{-1} \mathbf{F}^{-1}\left(\mathbf{a}_{\text {rec }}-\mathbf{b}_{\text {rec }}\right) .
\end{aligned}
$$

From substituting equation (A.18) and (A.19) in equation (A.9), we can derive

$$
\mathbf{b}_{\text {rec }}=\left(\mathbf{Z}+\mathbf{Z}_{0}\right)^{-1}\left(\mathbf{Z}-\mathbf{Z}_{0}^{*}\right) \mathbf{a}_{\text {rec }}+\frac{1}{2}\left(\mathbf{Z}+\mathbf{Z}_{0}\right)^{-1} \mathbf{F}^{-1} \mathbf{g}_{\mathbf{z}} \mathbf{A} \text {. }
$$
that

By comparing equations (A.20) and (A.14), one can see

$$
\begin{aligned}
& \mathbf{S}=\left(\mathbf{Z}+\mathbf{Z}_{0}\right)^{-1}\left(\mathbf{Z}-\mathbf{Z}_{0}^{*}\right), \\
& \mathbf{g}_{\mathbf{s}}=\frac{1}{2}\left(\mathbf{Z}+\mathbf{Z}_{0}\right)^{-1} \mathbf{F}^{-1} \mathbf{g}_{\mathbf{z}} .
\end{aligned}
$$

Substituting equations (A.13) and (A.5) in (A.22) gives

$$
\mathbf{g}_{\mathrm{s}}=\frac{2 \pi}{j \omega \mu_{0}}\left(\mathbf{Z}+\mathbf{Z}_{0}\right)^{-1} \mathbf{F}^{-1} \mathbf{Z} \mathbf{p}_{\mathbf{y}} .
$$

The voltage $\mathbf{V}_{\text {rec }}$ at the ports of a receiving array antenna terminated with loads $\mathbf{Z}_{0}$ can be calculated by substituting

$$
\mathbf{V}_{\text {rec }}=-\mathbf{Z}_{0} \mathbf{I}_{\text {rec }}
$$

in equation (A.15), (A.16), and (A.14):

$$
\begin{gathered}
\mathbf{a}_{\text {rec }}=0, \\
\mathbf{b}_{\text {rec }}=-\mathbf{F}\left(\mathbf{Z}_{0}+\mathbf{Z}_{0}^{*}\right) \mathbf{I}_{\text {rec }}=\mathbf{g}_{\mathbf{s}} \mathbf{A},
\end{gathered}
$$

and substituting (A.26) in (A.24),

$$
\mathbf{V}_{\text {rec }}=\mathbf{Z}_{0}\left(\mathbf{Z}_{0}+\mathbf{Z}_{0}^{*}\right)^{-1} \mathbf{F}^{-1} \mathbf{g}_{\mathbf{s}} \mathbf{A} \text {. }
$$

The minus sign in equation (A.24) is caused by the definition of the currents and voltages. A positive current is defined as a current flowing into the antenna port at the positive terminal. This means that the current flowing into a load is defined as a positive current if it is flowing out of the positive terminal.

\section{Data Availability}

The data used to support the findings of this study are available from the corresponding author upon request.

\section{Conflicts of Interest}

The authors declare that they have no conflicts of interest.

\section{References}

[1] S. J. Wijnholds, M. Arts, P. Bolli, P. di Ninni, and G. Virone, "Using embedded element patterns to improve aperture array calibration," in Proceedings of the 2019 International Conference on Electromagnetics in Advanced Applications (ICEAA), pp. 437-442, IEEE, Granada, Spain, September 2019.

[2] J. Hansen, Spherical Near-Field Antenna Measurements, Peter Peregrinus Ltd., London, UK, 1988.

[3] E. de Lera Acedo, N. Razavi-Ghods, D. G. Ovejéro, R. Sarkis, and C. Craeye, "Compact representation of the effects of mutual coupling in non-regular arrays devoted to the ska telescope," in Proceedings of the 2011 International Conference on Electromagnetics in Advanced Applications, pp. 390-393, IEEE, Turin, Italy, September 2011.

[4] H. Bui-Van, C. Craeye, N. Razavi-Ghods, and E. de Lera Acedo, "Correlation between ska1-low stations including mutual coupling," in Proceedings of the 2017 International Conference on Electromagnetics in Advanced Applications (ICEAA), pp. 1409-1411, IEEE, Verona, Italy, September 2017.

[5] K. F. Warnick, R. Maaskant, M. V. Ivashina, D. B. Davidson, and B. D. Jeffs, "High-sensitivity phased array receivers for radio astronomy," Proceedings of the IEEE, vol. 104, no. 3, pp. 607-622, 2016. 
[6] W. A. van Cappellen and L. Bakker, "Apertif: phased array feeds for the westerbork synthesis radio telescope," in Proceedings of 2010 IEEE International Symposium on Phased Array Systems and Technology, pp. 640-647, IEEE, Waltham, MA, USA, October 2010.

[7] B. Hut, R. H. van den Brink, and W. A. van Cappellen, "Status update on the system validation of apertif, the phased array feed system for the westerbork synthesis radio telescope," in Proceedings of the 2017 11th European Conference on Antennas and Propagation (EUCAP), pp. 1960-1961, IEEE, Paris, France, March 2017.

[8] A. Elsakka, T. A. H. Bressner, A. J. van den Biggelaar et al., "On the use of focal-plane arrays in $\mathrm{mm}$-wave $5 \mathrm{G}$ base stations," in Proceedings of the 12th European Conference on Antennas and Propagation (EUCAP 2018), pp. 1-4, IEEE, London, UK, April 2018.

[9] M. P. van Haarlem, M. W. Wise, A. W. Gunst et al., "The lowfrequency array," Astronomy \& Astrophysics, vol. 556, no. A2, 2013.

[10] M. James, “The generalised inverse," Mathematical Gazette, vol. 63, no. 425, pp. 181-185, 1978.

[11] J. Yu, V. A. Khlebnikov, and M.-H. Ka, "Wideband gratinglobe suppression by rotation of the phased array stations in the ska low-frequency sparse aperture array," IEEE Transactions on Antennas and Propagation, vol. 63, no. 9, pp. 3939-3946, Sep. 2015.

[12] https://altairhyperworks.com/product/FEKO.

[13] K. F. Warnick, D. B. Davidson, and D. Buck, "Embedded element pattern loading condition transformations for phased array modeling," IEEE Transactions on Antennas and Propagation, vol. 69, no. 3, pp. 1769-1774, 2021.

[14] G. Virone, P. Bolli, F. Paonessa et al., "Strong mutual coupling effects on lofar: modeling and in situ validation," IEEE Transactions on Antennas and Propagation, vol. 66, no. 5, pp. 2581-2588, 2018.

[15] A. T. de Hoop, "The N-port receiving antenna and its equivalent electrical network," Philips Research Reports, vol. 30, pp. 302-315, 1975.

[16] K. Kurokawa, "Power waves and the scattering matrix," IEEE Transactions on Microwave Theory and Techniques, vol. 13, no. 2, pp. 194-202, 1965. 